\title{
Description of Evaporation Curve for Liquid Metals by the Generalized Van-der-Waals-Berthelot Equation. Part II
}

\author{
Aleksandr Aleksandrovich Sobko
}

A M Prokhorov Academy of Engineering Sciences, Moscow 123557, Russian Federation

\begin{abstract}
In the present work it is shown that the generalized Van-der-Waals-Berthelot equation describes the evaporation curves (saturation curves) for alkali metals with good accuracy. This result is obtained on the basis of the calculations performed by the author for thermodynamic parameters of the saturation curves described by the generalized Van-der-Waals-Berthelot equation.
\end{abstract}

Key words: Van-der-Waals equation, Berthelot equation, generalized Van-der-Waals-Berthelot equation, evaporation curve, metals.

1. Calculation for Evaporation Curve for Van-der-Waals-Berthelot Gases and Comparison with Evaporation Curves for Alkali Metals

It was shown in Refs. [1, 2] that the evaporation curves for liquefied gases reside between those for Van-der-Waals and Berthelot gases. As can be seen from Fig. 1 the evaporation curves for liquid alkali metals reside between the evaporation curves for the Van-der-Waals and Berthelot gases as well. With variation of the parameter $\alpha$ in the generalized Van-der-Waals-Berthelot equation:

$$
P^{*}=\frac{8 T^{*}}{\left(3 V^{*}-1\right)}-\frac{3}{T^{* \alpha} V^{* 2}}
$$

one can achieve good agreement between the experimental evaporation curves for alkali metals and the evaporation curves described by the generalized Van-der-Waals-Berthelot equation.

The author calculated the evaporation curves for the Van-der-Waals-Berthelot gas with $\alpha \in$ [0.0-1.0] and the step of 0.1 . The equal area rule (Maxwell's rule) was used in the calculations. Additionally, it was proved in Refs. [1, 2] that the derivative at the critical

Corresponding author: Aleksandr Aleksandrovich Sobko, Dr., academician, research fields: thermodynamics, statistical physics, quantum mechanics. point is $\left(P_{T^{*}}^{*^{\prime}}\right)_{c . t}=\left(\frac{d P^{*}}{d T^{*}}\right)_{c . t}=3 \alpha+4$ for the generalized Van-der-Waals-Berthelot equation.

The evaporation curves for alkali metals and the Van-der-Waals-Berthelot gas are provided and analyzed below. All experimental data on the evaporation curves for alkali metals were taken from the handbook [3].

\section{Evaporation Curves for Alkali Metals}

\subsection{Analysis of Evaporation Curves for Lithium}

The evaporation curves for lithium and the Van-der-Waals-Berthelot gas with the parameter $\alpha=0.7$ are given in Fig. 2. The evaporation curve for lithium and the evaporation curve for the Van-der-Waals-Berthelot gas are in good agreement as can be seen from the figure.

\subsection{Analysis of Evaporation Curve for Sodium}

Two evaporation curves for sodium and the evaporation curve for the Van-der-Waals-Berthelot gas are presented in Fig. 3. There are two evaporation curves for sodium as the data on the critical pressure values for sodium taken from two different sources reveal significant difference. According to the values from the handbook [3] bar, while [4] provides bar. Corresponding critical temperatures are almost equal 


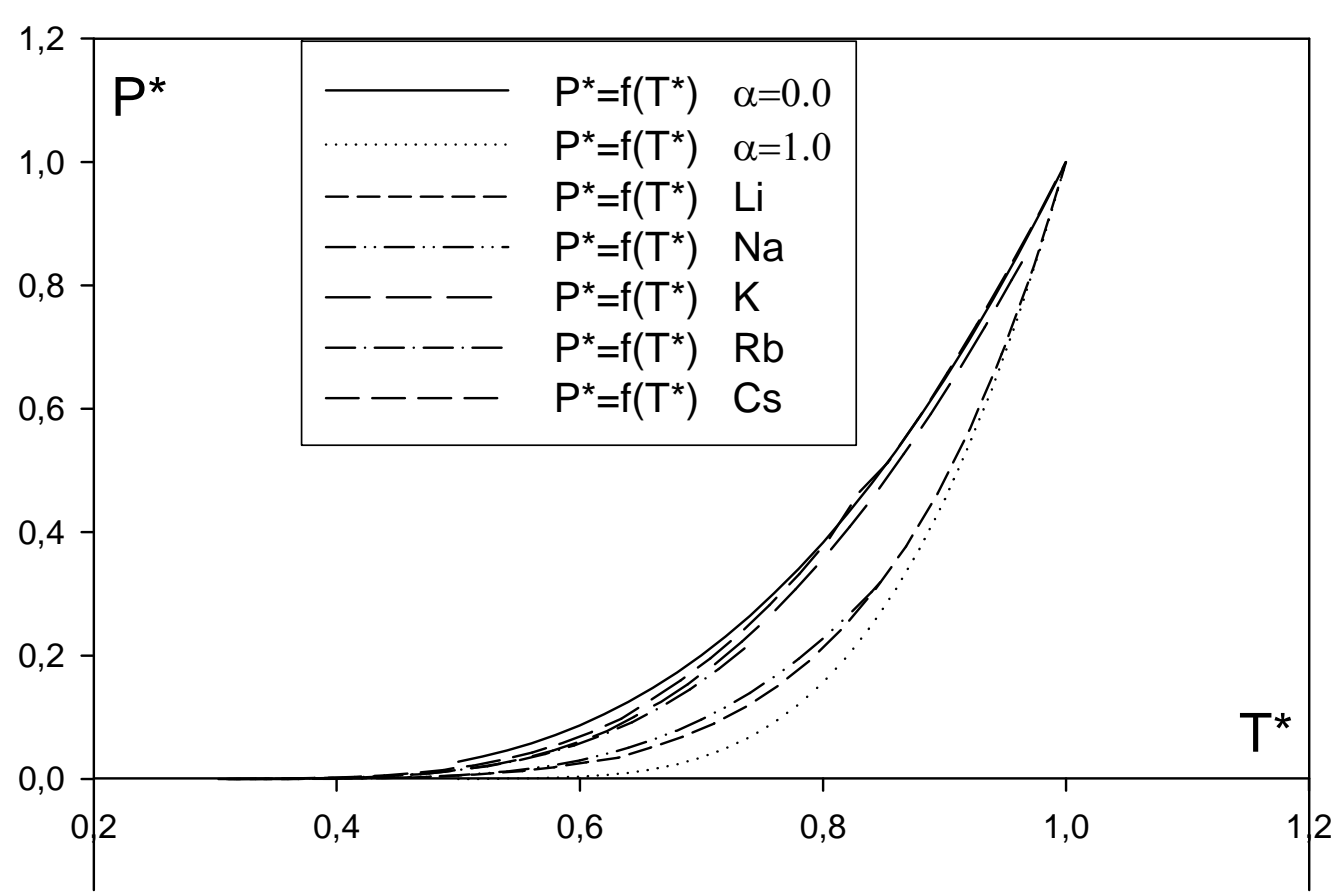

Fig. 1 Equilibrium lines for Van-der-Waals and Berthelot gases and equilibrium lines for liquid alkali metals.

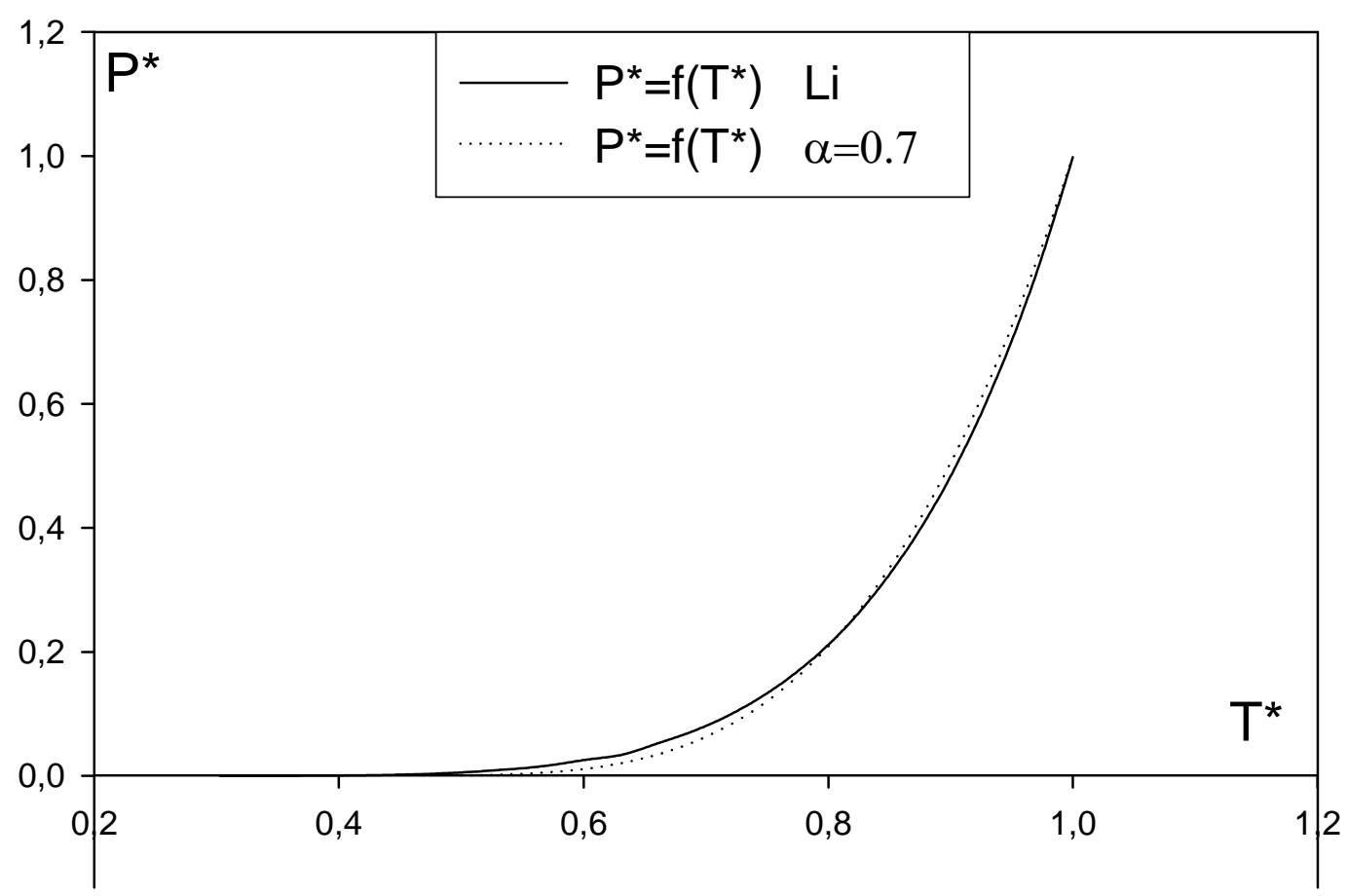

Fig. 2 Equilibrium lines for lithium and Van-der-Waals-Berthelot gas. Derivatives at critical point are $\left(P_{T^{*}}^{*^{\prime}}\right)_{c . e x}=6.33$ and $\left(P_{T^{*}}^{*^{\prime}}\right)_{c . t}=6.1$, respectively, with error of $\delta_{L i}=3.64 \%$. 


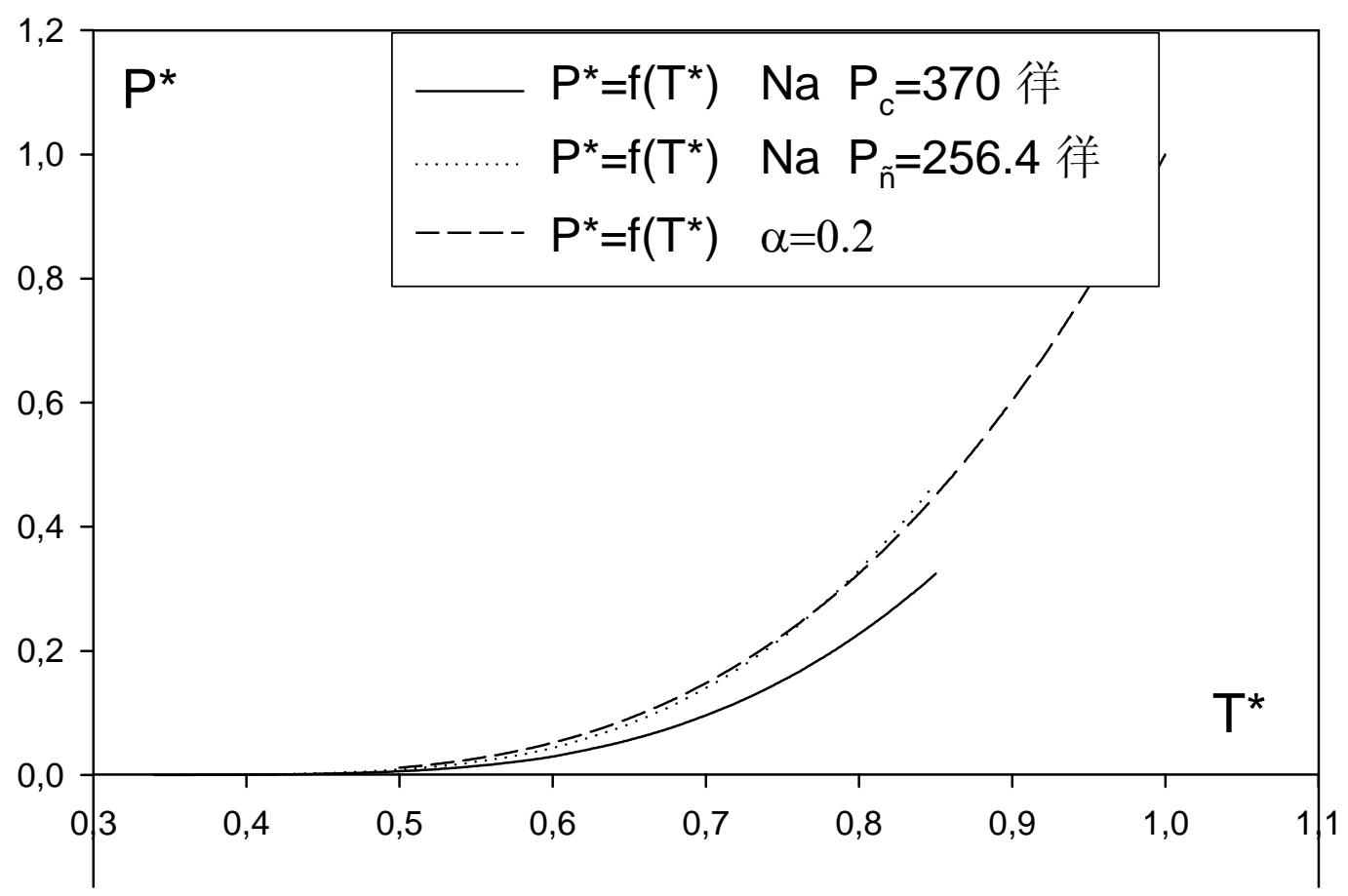

Fig. 3 Equilibrium lines for sodium and Van-der-Waals-Berthelot gas.

but the critical pressure values are significantly different. Therefore, Fig. 2 contains two evaporation curves for sodium for the given values of temperature $T^{*}$ and pressure $P^{*}$. These curves were calculated with the use of the data from Refs. [3] and [5]. The data given in Ref. [4] are more reliable as this handbook was published in 1990 while Ref. [3] was published in 1972. Indeed, the evaporation curve obtained with $P_{c}=256.4$ bar is in good agreement with the evaporation curve for the Van-der-Waals-Berthelot gas with the parameter $\alpha=0.2$. The evaporation curve for sodium obtained with the critical pressure value of $P_{c}=370$ bar does not match the evaporation curve for the Van-der-Waals-Berthelot gas for any given value of the parameter $\alpha$.

The experimental data given in Ref. [3] on the evaporation curve for sodium do not approach the critical point by far. Thus, it is impossible to calculate the $\left(P_{T^{*}}^{*^{\prime}}\right)_{\text {c.ex }}$ derivative at the critical point. The derivatives at the ending point of the experimental data are $\left(P_{T^{*}}^{*^{\prime}}\right)_{e x}=2.13$ for $P_{c}=370$ bar and
$\left(P_{T^{*}}^{*^{\prime}}\right)_{e x}=3.08$ for $P_{c}=256.4$ bar. These derivative values are lower than the theoretically predicted value of $\left(P_{T^{*}}^{*^{\prime}}\right)_{c . t}=4.6$.

\subsection{Analysis of Evaporation Curve for Potassium}

The evaporation curves for potassium and the Van-der-Waals-Berthelot gas with the parameter $\alpha=0.1$ are given in Fig. 4 . The evaporation curve for potassium and the evaporation curve for the Van-der-Waals-Berthelot gas are in good agreement as can be seen from the figure. The derivatives at the critical point are $\left(P_{T^{*}}^{*^{\prime}}\right)_{\text {c.ex }}=4.50$ and $\left(P_{T^{*}}^{*^{\prime}}\right)_{c, t}=4.30$, respectively, with the error of $\delta_{K}=4.44 \%$.

\subsection{Analysis of Evaporation Curve for Rubidium}

The evaporation curve for rubidium and two evaporation curves for the Van-der-Waals-Berthelot gas with the parameters $\alpha=0.1$ and $\alpha=0.2$ are given in Fig. 5. The evaporation curve for rubidium resides between the evaporation curves for the 


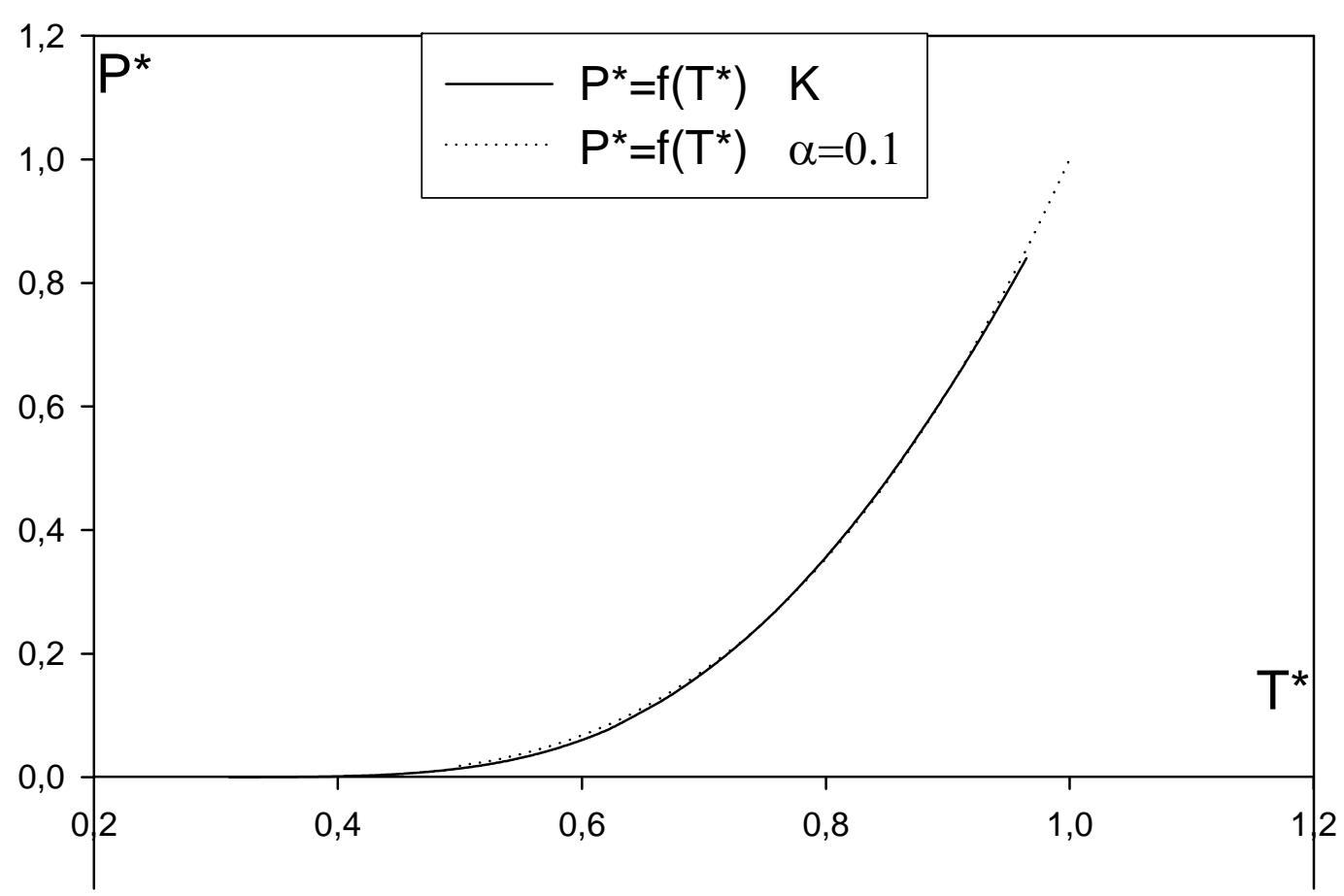

Fig. 4 Equilibrium lines for potassium and Van-der-Waals-Berthelot gas.

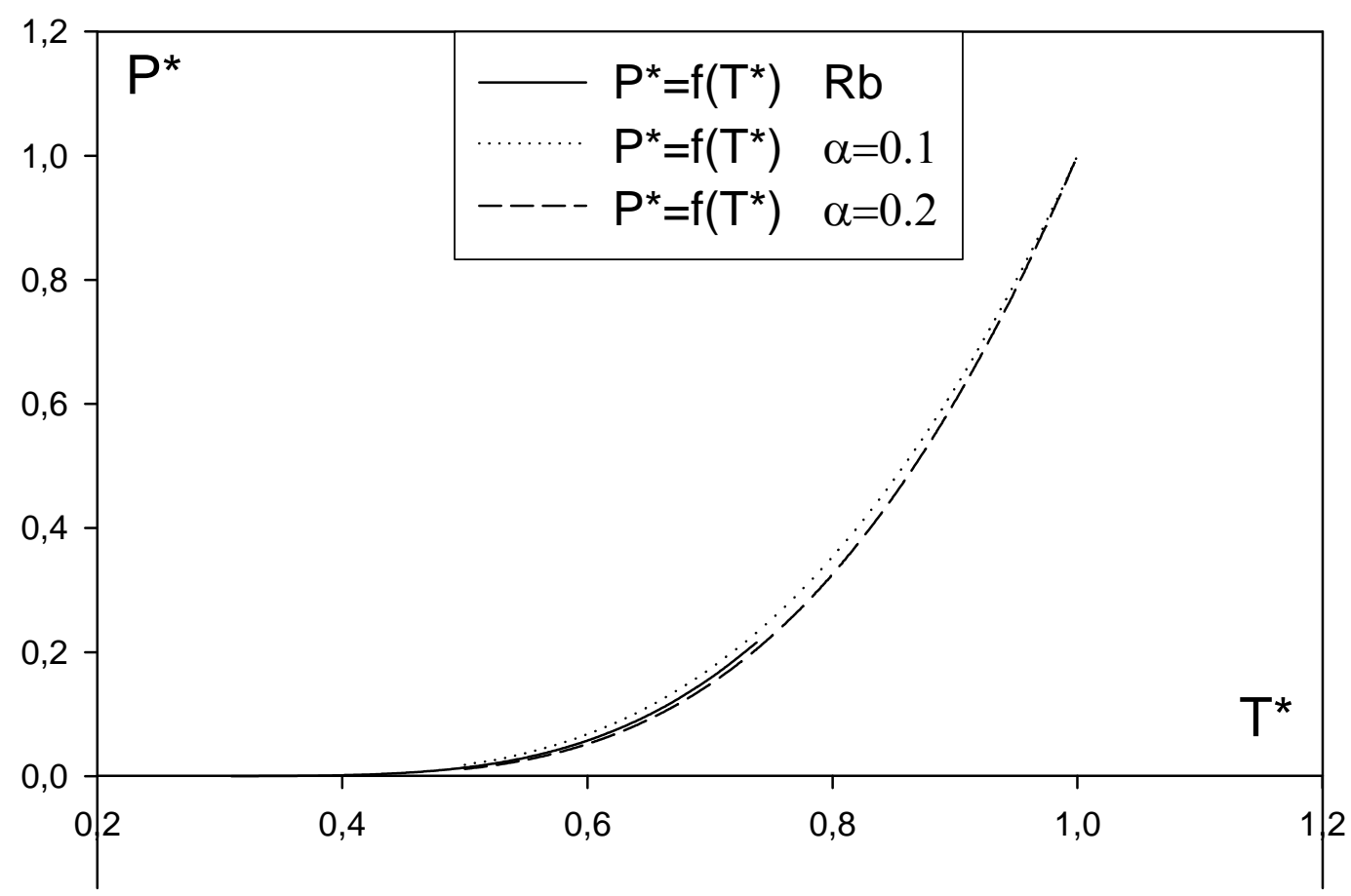

Fig. 5 Equilibrium lines for rubidium and Van-der-Waals-Berthelot gas. 


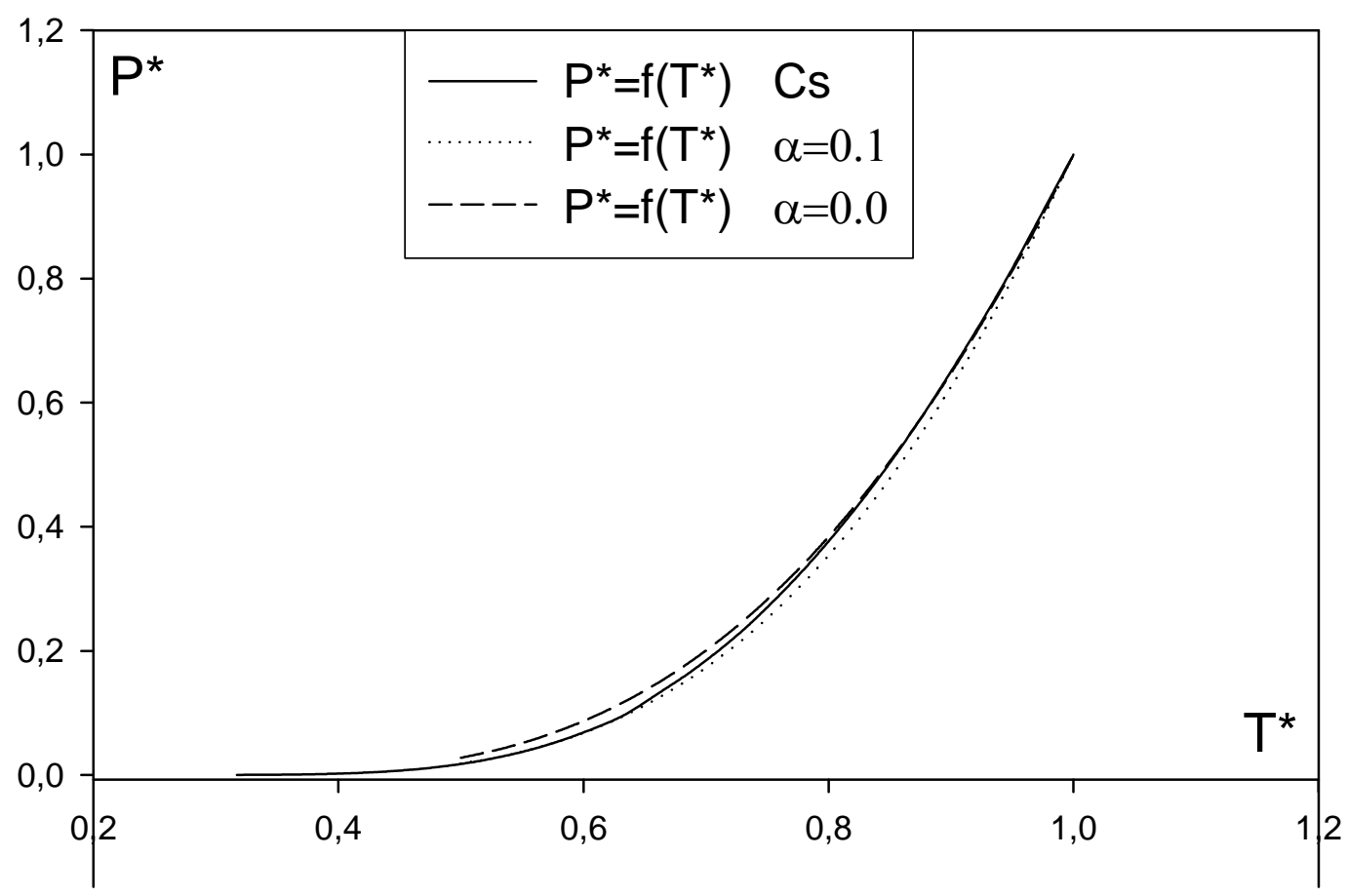

Fig. 6 Equilibrium lines for cesium and Van-der-Waals-Berthelot gas.

Van-der-Waals-Berthelot gas with the parameter $\alpha$ specified in the range of $0.1<\alpha<0.2$ as can be seen from the figure. Unfortunately, the experimental data on the evaporation curve are far from the critical point. The derivative at the ending point of the experimental data is $\left(P_{T^{*}}^{*^{\prime}}\right)_{e x}=1.5$. This value is substantially lower than the theoretically predicted value of the derivative at the critical point $\left(P_{T^{*}}^{*^{\prime}}\right)_{c . t}=4.30 \div 4.60$.

\subsection{Analysis of Evaporation Curve for Cesium}

The evaporation curve for cesium and two evaporation curves for the Van-der-Waals-Berthelot gas with the parameters $\alpha=0.0$ and $\alpha=0.1$ are given in Fig. 6. The evaporation curve for cesium resides between the evaporation curves for the Van-der-Waals-Berthelot gas. This evaporation curve can be described by the Van-der-Waals-Berthelot equation with the parameter $\alpha$ specified in the range of $0.0<\alpha<0.1$.
The experimental value of the derivative is $\left(P_{T^{*}}^{*^{\prime}}\right)_{e x}=3.75$; the theoretically predicted value of the derivative at the critical point is $\left(P_{T^{*}}^{*^{\prime}}\right)_{c . t}=4.0$ with the error of $\delta_{C s}=6.26 \%$.

\section{Conclusions}

(1) The results given above demonstrate that the generalized Van-der-Waals-Berthelot equation describes the evaporation curves for alkali metals with good accuracy (about several percent).

(2) Good agreement between the experimental values and theoretical predictions $\left(P_{T^{*}}^{*^{\prime}}\right)_{c . t}=3 \alpha+4$ was obtained for the derivatives at the critical point, provided that the experimental values of $T^{*}$ and $P^{*}$ were known in the vicinity of the critical point.

(3) The agreement between the calculated values and experimental data can be further improved with specifying the value of the parameter $\alpha$ with two-decimal accuracy rather than one-decimal accuracy while calculating the evaporation curves for the Van-der-Waals-Berthelot gas. 


\section{References}

[1] Sobko, A. A. 2008. "Generalized van der Waals-Berthelot Equation of State.” Doklady Physics 53 (8): 416.

[2] Sobko, A. A. 2014. "Description of Evaporation Curve by the Generalized Van-der-Waals-Berthelot Equation.
Part I.” Journal of Physical Science and Application 4 (8): 524.

[3] Vargaftik, N. B. 1972. Handbook on Thermal Properties of Gases and Liquids. Moscow, Nauka (in Russian).

[4] Anisimov, M. A., Rabinovich, V. A., and Sychev, V. V. 1990. Thermodynamics of the Critical State of Individual Materials. Moscow, Energoatomizdat (in Russian). 